\title{
The Unconditional Instability of Inflow-Dependent Boundary Conditions in Difference Approximations to Hyperbolic Systems
}

\author{
By Eitan Tadmor*
}

\begin{abstract}
It is well known that for a mixed initial-boundary hyberbolic system to be well-defined it is necessary to impose additional boundary conditions only on the inflow eigenspace of the problem. We prove the discrete analogue of the above concerning difference approximations to such a system; that is, imposing numerical boundary conditions which are at least zeroth-order accurate with an inflow part of the interior equations leads to unconditional instability.
\end{abstract}

1. Introduction. We consider the first-order hyperbolic system

$$
\frac{\partial u}{\partial t}=\mathbf{P} u+F, \quad \mathbf{P} \equiv A \frac{\partial}{\partial x}+E
$$

in the quarter plane $x, t \geqslant 0$, subject to initial conditions

$$
\left.u\right|_{t=0}=f(x), \quad x \geqslant 0 .
$$

Here $u \equiv u(x, t)$ is the $N$-dimensional vector of unknowns; the $N$-dimensional coefficient matrices $A \equiv A(x, t), E \equiv E(x, t)$ and inhomogenous vector functions $F \equiv F(x, t)$ smoothly depend on $(x, t)$, and by hyperbolicity we mean that $A$ is similar to a real diagonal $\Lambda$

$$
\begin{gathered}
T A T^{-1}=\Lambda \equiv \operatorname{diag}\left(\Lambda^{-}, \Lambda^{+}\right), \\
\Lambda^{-} \equiv \operatorname{diag}\left(\lambda_{l}^{-}, \ldots, \lambda_{l}^{-}\right)<0<\Lambda^{+} \equiv \operatorname{diag}\left(\lambda_{l+1}^{+}, \ldots, \lambda_{N}^{+}\right) .
\end{gathered}
$$

The principal part of system (1.1a)-rewritten in its characteristic form $\partial \tilde{u} / \partial t=$ $\Lambda \partial \tilde{u} / \partial t+\tilde{F}, \sim$ denoting multiplication by $T$ on the left-asserts that the characteristic variables $\tilde{u}$ are uniquely determined along the curves $\dot{x}_{j}-\lambda_{j}^{ \pm}=0$. The last $N-l$ of these characteristic curves carry $N-l$ pieces of information-these are the outflow components $\tilde{u}^{+} \equiv\left(\tilde{u}^{(l+1)}, \ldots, \tilde{u}^{(N)}\right)^{T}$ impinging on the boundary $x=0$ from the right. For system (1.1) to be uniquely solvable therefore, exactly $l$ additional pieces of information must be provided at $x=0$

$$
\left.\mathbf{B} u\right|_{x=0}=G, \quad \operatorname{rank}[\mathbf{B}]=l .
$$

Received May 10, 1982.

1980 Mathematics Subject Classification. Primary 65N10, 65M10.

*Work was supported in part by NASA Contract No. NAS 1-17070 while the author was in residence at ICASE, NASA Langley Research Center, Hampton, VA 23665. Part of the research reported in this paper was carried out while the author was a Bateman Research Instructor in the Department of Applied Mathematics, California Institute of Technology, Pasadena, California. 
The requirement for the boundary conditions to be on top of the predetermined outflow components, can be expressed as follows (Hersh [7]):

Let $\Phi^{-}$denote the negative eigenspace spanned by the eigenvectors $\left\{\phi_{j}^{-}\right\}_{j=1}^{l}$ associated with the negative eigenvalues $\left\{\lambda_{j}^{-}\right\}_{j=1}^{l}$. Then, for all nontrivial vectors $\phi^{-}$ in $\Phi^{-}$, we must have

$$
\mathbf{B} \phi^{-} \neq 0, \quad 0 \neq \phi^{-} \in \Phi^{-} .
$$

Condition (1.2b) merely says that, at the boundary $x=0$, it is necessary to provide extra information for the inflow components $\tilde{u}^{-} \equiv\left(\tilde{u}^{(1)}, \ldots, \tilde{u}^{(l)}\right)^{T}$, in addition to that governed in the interior by the differential system (1.1a). In other words, if the boundary conditions (1.2a) are inflow-dependent in the sense that equality takes place in (1.2b) for some nontrivial $\phi_{*}^{-}$in $\Phi^{-}$,

$$
\left.\mathbf{B} \phi_{*}^{-}\right|_{x=0}=0, \quad 0 \neq \phi_{*}^{-} \in \Phi^{-},
$$

then the problem (1.1)-(1.2) is ill-posed. In the one-dimensional case, conditions (1.2a)-(1.2b) amount to the standard reflection $\tilde{u}^{-}=\tilde{\mathbf{B}} \tilde{u}^{+}+\tilde{G}$, implying, in particular, that the requirement $(1.2 b)$ is necessary as well as sufficient for the problem to be well-posed. In the multi-dimensional case to be discussed later, however, requirement (1.2b) with appropriately defined $\Phi^{-}$serves only as a necessary condition for well-posedness (e.g. [11, Chapter 15]).

In this paper we study difference approximations to system (1.1)-(1.2) consisting of an interior scheme

$$
\left[I+\Delta t \mathscr{P}_{\Delta x}\right] v(t+\Delta t)=\left[I+\Delta t P_{\Delta x}\right] v(t)+\Delta t F_{\Delta x}
$$

together with boundary conditions

$$
\left[\Re_{\Delta x}\right] v(t+\Delta t)=\left[B_{\Delta x}\right] v(t)+\Delta t G_{\Delta x} \text {. }
$$

In analogy with (1.3) we say that the boundary conditions (1.4b) are inflow-dependent if they are at least zeroth-order accurate with an inflow part of the system; that is, if for some nontrivial $\phi_{*}^{-}$in $\Phi^{-}$we have

$$
\left[\mathscr{B}_{\Delta x}-B_{\Delta x}\right] \phi_{*}^{-}=0, \quad 0 \neq \phi_{*}^{-} \in \Phi^{-} .
$$

Our aim is to show that such inflow-dependent boundary conditions render the overall computation unstable-a discrete analogue of the above-mentioned ill-posedness occurring in the differential problem.

We note that boundary conditions based on prescribing given data are not included in the above inflow-dependent class since such a recipe is not even zeroth-order accurate.

The unconditional ill-posedness ( $\leftrightarrow$ instability) in both the differential and differenced cases is of course due to the independence of the inflow boundary values on the interior equations. Unlike the differential problem however, the discrete symbol is lacking the homogeneity property which is the technical key for showing ill-posedness [7]. Yet, by consistency, one can focus his attention on the behavior of the difference symbol near the origin where it approximates the differential one. After setting the precise framework for our discussion in the next section, proof of the main result along these lines is carried out in Section 3. We conclude with examples and further clarifying remarks in Section 4. Finally, we note that the above 
result, though its proof is formulated to keep the notations simple in the one-dimensional setup, is carried over, as will be later indicated, for the multi-dimensional problem as well.

2. Statement of Main Result. We would like to solve (1.1)-(1.2) by difference approximations. In order to do so, we introduce a mesh size $\Delta x>0$ and a time-step $\Delta t>0$ such that $\lambda \equiv \Delta t / \Delta x=$ constant. Using the standard notation $v_{\nu}(t) \equiv$ $v(\nu \Delta x, t)$, we approximate (1.1 a) by a consistent solvable two-step interior scheme of the form

$$
\sum_{j=-r}^{p} \mathbb{Q}_{j}\left(x_{\nu}, \Delta x\right) v_{\nu+j}(t+\Delta t)=\sum_{j=-r}^{p} A_{j}\left(x_{\nu}, \Delta x\right) v_{\nu+j}(t)+\Delta t H_{\nu}(t)
$$

$$
\nu \geqslant r
$$

Starting with the initial data

$$
v_{\nu}(t=0)=f_{\nu}, \quad \nu \geqslant 0,
$$

the scheme (2.1a) augmented by solvable boundary conditions

$$
\begin{aligned}
\sum_{j=0}^{q} \Re_{j \nu}\left(x_{\nu}, \Delta x\right) v_{j}(t+\Delta t)=\sum_{j=0}^{q} B_{j \nu}\left(x_{\nu}, \Delta x\right) v_{j}(t)+\Delta t H_{\nu}(t), & \\
\nu & =0,1, \ldots, r-1,
\end{aligned}
$$

is then used to advance in time.

To guarantee the convergence of the discrete solution $v_{\nu}(t)$ to the exact one $u(x, t)$ as the mesh being refined, $\Delta x, \Delta t \rightarrow 0$, we have to verify that the difference approximation (2.1) is stable [3]; that is, we want small perturbations of either the initial or the inhomogeneous data not to excite the principal part of our computation, but rather to have only a small comparable effect. We refer the reader to [5] for the precise definitions of stability with respect to either the initial data [5, Definition 3.1], or the inhomogeneous data [5, Definitions 3.2 and 3.3].

We start by making the natural

Assumption ([5, Assumption 5.3]). The interior scheme (2.1a) is $l_{2}$-stable when applied for the Cauchy problem $-\infty<\nu<\infty$.**

Our task is, therefore, to determine whether the assumed interior stability is deteriorating due to the introduction of the boundary conditions (2.1c). We claim that this is indeed the situation for inflow-dependent boundary conditions, see (1.5). The main result of the paper thus reads

THEOREM. If the boundary conditions (2.1c) are inflow-dependent in the sense that for some nontrivial $\phi_{*}^{-}$in the inflow (= negative) eigenspace $\Phi^{-}$we have

$$
\sum_{j=0}^{q}\left[\mathscr{B}_{j \nu}(0,0)-B_{j \nu}(0,0)\right] \phi_{*}^{-}=0, \quad \nu=0,1, \ldots, r-1,
$$

then the overall approximation (2.1) is unstable.

\footnotetext{
${ }^{*}$ Hereafter $l_{2}$ denotes the space of all grid functions $\left\{\chi_{\nu}\right\}$ satisfying $\Sigma_{\nu}\left|\chi_{\nu}\right|^{2}<\infty$, the summation being taken over all relevant grid points.
} 
The above result is the exact discrete analogue of the ill-posedness inferred in the differential problem by (1.3). In the special case of horizontal boundary extrapolation, it was first stated by Kreiss [8] for the one-dimensional scalar problem and proved by Burns [1] for systems. The somewhat simpler extension given here treats general boundary conditions augmenting any two-leveled interior scheme. Furthermore, we remove the scalar-like restriction imposed in [1, Assumption 3.2], requiring $Q_{j}, A_{j}$ to be polynomials in $A$; so, by using standard reblocking and Fourier transforming of tangential derivatives, the above theorem is valid as well for multi-leveled schemes approximating the multi-dimensional problem.

The proof of the theorem given in the next section, proceeds by showing step by step the unconditional instability with respect to each of the stability definitions [5, Definitions 3.1, 3.2 and 3.3]. In particular, we give an explicit estimate of the unstable growth of the discrete solution for dissipative schemes; more precisely, we show in such a case that if the inflow boundary conditions are $s$-order accurate, see (3.10), then after $n$ time steps the $l_{2}$-norm of the solution exceeds $\sim n^{(s+1 / 2)}-$ take $s=0$ to coincide with the $l_{2}$-instability [5, Definition 3.1] stated in the main theorem. Such a weak polynomial instability is nevertheless rejected as being inappropriate due to possible reflections at the other (right) boundary which may result into the untolerable exponential instability [11, Chapter 17].

We close this section with the following concrete example. Consider any standard 5-point interior scheme approximating the linearized inviscid one-dimensional gasdynamics equations (low-order terms due to linearization are neglected)

$$
\frac{\partial u}{\partial t}+A \frac{\partial u}{\partial x}=F, \quad x, t \geqslant 0
$$

here $u=(\rho, U, p)^{T}$ are the density velocity and pressure respectively, $F$, stands for the external forces and

$$
A=\left[\begin{array}{ccc}
U_{s} & \rho_{s} & 0 \\
0 & U_{s} & 1 / \rho_{s} \\
0 & \gamma p_{s} & U_{s}
\end{array}\right], \quad \gamma=\text { ratio of specific heats, }
$$

with $\left(\rho_{s}, U_{s}, p_{s}\right)^{T}$ denoting the corresponding state we linearize about. We are interested in the subsonic inflow case, $0<U_{s}<c_{s}, c_{s} \equiv \sqrt{\gamma p_{s} / \rho_{s}}$, where a twodimensional inflow eigenspace is to be determined at $\left(x_{1}, t+\Delta t\right)$ and-in case exact inflow conditions are not known-at $\left(x_{0}, t+\Delta t\right)$ as well. According to the above theorem, any attempt to calculate the missing values using at least zeroth-order accurate conditions for either $\tilde{u}_{1}^{-}=c_{s}^{2} \rho-p, \tilde{u}_{2}^{-}=\rho_{s} c_{s} U+p$, or any combination of them, will result in instability. In other words, the computation of these missing values requires additional information which is not to be extracted solely from the differential equations in the interior, (1.1a). One possibility, of course, is to take into account the differential boundary conditions (1.2), if known. A general detailed procedure along these lines, to extract the additional information to any desired degree of accuracy, is described in [2].

3. Unconditional Instability. By the nature of our negative result, it is sufficient to restrict attention to the principal part of the approximation localized at $x=0$. Indeed, by introducing appropriate boundary cut-off functions, one finds that it is 
the constant coefficient cases

$$
\mathbb{Q}_{j} \equiv \mathbb{Q}_{j}(0,0), \quad A_{j} \equiv A_{j}(0,0), \quad \mathscr{B}_{j \nu} \equiv \mathscr{B}_{j \nu}(0,0), \quad B_{j \nu} \equiv B_{j \nu}(0,0),
$$

which determine the stability properties overall.

Let $\mathcal{L}_{\Delta x}$ stand for the constant coefficient evolution operator which represents advancing of one time-step with approximation (2.1a), (2.1c). Then, starting with the initial data $f=\left\{f_{\nu}\right\}_{\nu=0}^{\infty}$, our approximate solution $v=\left\{v_{\nu}\right\}_{\nu=0}^{\infty}$ after $n$ such time-steps is given by

$$
v(t=n \cdot \Delta t)=\mathfrak{e}_{\Delta x}^{n} f .
$$

Employing the Cauchy integral representation, it can also be rewritten as

$$
v_{\nu}(t=n \cdot \Delta t)=\frac{1}{2 \pi i} \int_{\Gamma} z^{n} \psi_{\nu}(z) d z, \quad \nu=0,1, \ldots,
$$

with the contour $\Gamma$ enclosing the spectrum of $L_{\Delta x}$, and the vector grid function $\psi(z)=\left\{\psi_{\nu}(z)\right\}_{\nu=0}^{\infty}$ determined by the resolvent equation

$$
\left[z I-\varrho_{\Delta x}\right] \psi(z)=f, \quad\left\{\psi_{\nu}(z)\right\}_{\nu=0}^{\infty} \in l_{2}(0, \infty) .
$$

We continue with the construction of a general solution for (3.1c), in the case of vanishing interior initial data, $f_{\nu_{\nu>r}}=0$, e.g. [4, Section 5], [8, Section 2].

The evolution operator $L_{\Delta x}$ appearing in the resolvent equation (3.1c) consists of two parts-corresponding to the basic scheme (2.1a) we have in the interior

$$
\sum_{j=-r}^{p}\left(z \mathbb{Q}_{j}-A_{j}\right) \psi_{\nu+j}(z)=0, \quad \nu=r, r+1, \ldots,
$$

augmented at the boundary by, see (2.1c),

$$
\sum_{j=0}^{q}\left(z \mathscr{G}_{j \nu}-B_{j \nu}\right) \psi_{j}(z)=f_{\nu}, \quad \nu=0,1, \ldots, r-1 .
$$

Equation (3.2a) is an ordinary difference equation with constant matrix coefficients, whose most general $l_{2}$-bounded solution is given by [12]

$$
\psi_{k}(z)=X(z) L^{k}(z) \boldsymbol{\sigma}, \quad k=0,1, \ldots,|z|>1 .
$$

Here $L(z)$ is an $N r$-dimensional matrix which consists of the power-bounded Jordan cells associated with the characteristic eigenvalue problem

$$
\operatorname{Det}\left[\sum_{j=-r}^{p}\left(z Q_{j}-A_{j}\right) \kappa^{j}\right]=0 \text {; }
$$

the $N \times N r$ rectangular matrix $X(z)$ consists of $N r$ column vectors $\left\{\phi_{m}(z)\right\}_{m=1}^{N r}$ which are the corresponding $N$-dimensional Jordan chains, and finally, $\sigma$ is an $\mathrm{Nr}$-dimensional vector to be determined by the additional $\mathrm{Nr}$ boundary conditions (3.2b): inserting (3.3) into (3.2b) we end up with the system of $\mathrm{Nr}$ equations

(3.5a) $\quad D(z) \boldsymbol{\sigma}=\mathbf{f}, \quad D(z)=\left[\begin{array}{c}D_{[0]}(z) \\ D_{[1]}(z) \\ \vdots \\ D_{[r-1]}(z)\end{array}\right], \quad \mathbf{f}=\left(f_{0}, \ldots, f_{r-1}\right)^{T}$, 
where $D_{[\nu]}(z)$ are the $N \times N r$-dimensional blocks

$$
D_{[\nu]}(z) \equiv \sum_{j=0}^{q}\left(z \Re_{j \nu}-B_{j \nu}\right) X(z) L^{j}(z), \quad \nu=0,1, \ldots, r-1 .
$$

The key of the instability proof ahead lies in studying the behavior of the solution for the resolvent equation, $\psi(z)$, in the neighborhood of $z=1$ (or, as already has been mentioned as the same thing - the dual variables near the origin). We will show that our boundary conditions being inflow-dependent, (2.2), imply the singularity of the solution at $z=1$, which in turn leads to unconditional instabilty. First, let us recall some further information about the solution $\psi(z)$, just constructed in (3.3), to be used later on.

Without loss of generality, the matrix $L(z)$ in the neighborhood of $z=1$ is of the form [5, Theorem 9.1]

$$
L(z)=\left[\begin{array}{cc}
L_{-}(z) & 0 \\
0 & L_{0}(z)
\end{array}\right]
$$

where the upper-left $l$-dimensional block, $L_{-}(z)$, represents those modes consistent with the inflow part of the differential equation [9, Lemma 7]

$$
L_{-}(z)=I+\left[\lambda \Lambda^{-}\right]^{-1}(z-1)+\vartheta(z-1)^{2},
$$

while in the lower-right $(\mathrm{Nr}-\mathrm{l})$-dimensional block, $L_{0}(z)$, we regroup the parasitic modes which eventually will be damped away from the boundary

$$
L_{0}^{*}(z) L_{0}(z) \leqslant(1-\delta) \cdot I, \quad \delta>0 .
$$

Next, let $X_{-}(z)$ denote the first $l$ column vectors in $X(z), \phi_{m}(z), m=1,2, \ldots, l$,

$$
X(z)=\left[X_{-}(z), X_{0}(z)\right]
$$

Inserting into (3.4) their corresponding eigenvalues from (3.6b), $\kappa_{m}(z)=1+$ $(z-1) /\left(\lambda \lambda_{m}^{-}\right)+\vartheta(z-1)^{2}$ and using the consistency of the interior scheme which amounts to the standard

$$
\sum_{j=-r}^{p}\left[Q_{j}-A_{j}\right]=\sum_{j=-r}^{p}\left[j\left(Q_{j}-A_{j}\right)+\lambda \mathbb{Q}_{j} A\right]=0
$$

we arrive at

$$
(z-1) \cdot \sum_{j=-r}^{p} Q_{j}\left[I+z \lambda_{m}^{-1} A\right] \phi_{m}(z)=O(z-1)^{2} .
$$

By the solvability of the interior scheme $\left.\Sigma Q_{j} e^{i j \theta}\right|_{\theta=0}=\Sigma Q_{j}$ is nonsingular; dividing by $(z-1) \cdot \Sigma \mathbb{Q}_{i}$, we conclude

$$
X_{-}(z)=X_{-}(1)+\vartheta(z-1),
$$

where the $l$ column vectors $\phi_{m}(1), m=1,2, \ldots, l$, which form $X_{-}(1)$, coincide with the inflow eigenvectors $\phi_{m}^{-}$of $A$ associated with its negative eigenvalues $\lambda_{m}^{-}<0$.

Proof of Main Theorem. We claim that the matrix $D(z)$ in (3.5a) is singular at $z=1$. Indeed, referring back to our inflow-dependence assumption, we recall the existence of $\phi_{*}^{-}$in the inflow eigenspace $\Phi^{-}$satisfying (2.2); that is, $\phi_{*}^{-}$belongs to the 
range of $X_{-}(1)$. Choose $\tau$ to be the $N r$-dimensional vector whose first $l$ components - denoted here by $\tau_{-}$- are uniquely determined as the solution of

$$
X_{-}(1) \tau_{-}=\phi_{*}^{-} \text {, }
$$

and its remaining $\mathrm{Nr}-l$ components are taken to be zero,

$$
\boldsymbol{\tau}=\left(\boldsymbol{\tau}_{-}, \mathbf{0}_{N r-l}\right)^{T}, \quad \boldsymbol{\tau} \neq 0 .
$$

Inserting this choice of $\tau$ into (3.5b), one finds on account of (3.6a) and (3.7a)

$$
[D(z) \tau]_{[\nu]}=\sum_{j=0}^{q}\left(z \mathscr{B}_{j \nu}-B_{j \nu}\right) X_{-}(z) L_{-}^{j}(z) \tau_{-}, \quad 0 \leqslant \nu \leqslant r-1 .
$$

Abbreviate the right-hand side of (3.9a) by $R_{[\nu]}(z)$. Making use of (3.6b) and (3.7b), then the inflow-dependence assumed in (2.2) implies for $\tau_{-}$chosen by (3.8a)

$$
\begin{aligned}
R_{[\nu]}(1) & =\sum_{j=0}^{q}\left(\circlearrowleft B_{j \nu}-B_{j \nu}\right) X_{-}(1) \cdot I \cdot \tau_{-} \\
& =\sum_{j=0}^{q}\left(\mathscr{B}_{j \nu}-B_{j \nu}\right) \phi_{*}^{-}=0, \quad 0 \leqslant \nu \leqslant r-1 .
\end{aligned}
$$

Thus, according to the terminology of Theorem 5.1 in [5], it was shown that $z=1$ is a generalized eigenvalue, and hence the approximation (2.1a), (2.1c) is unstable with respect to [5, Definition 3.3]. Furthermore, we note that the more accurate the boundary conditions are in the inflow direction $\phi_{*}^{-}$, say accurate of order $s-1 \geqslant 0$,

$$
|\mathbf{R}(z)|=\theta(z-1)^{s}, \quad \mathbf{R} \equiv\left(R_{[0]}, \ldots, R_{[r-1]}\right)^{T},
$$

the worse is the unstable (singular) behavior at $z=1$, in agreement with $[1$, Section 5],

$$
\operatorname{Det}[D(z)]=\vartheta(z-1)^{s}, \quad s \geqslant 1 .
$$

As shown by the counterexample in Section 4 below (see also [14, Section 5]), the existence of a (possibly generalized) eigenvalue on the unit circle does not necessarily imply instability with respect to either stability definitions 3.2 or 3.3 in [5]. We next turn to show, however, that the generalized eigenvalue $z=1$ in our case will always induce instability with respect to both, the reason being that this generalized eigenvalue is originating from the inflow part of the problem. To see that, we use (3.10) to make a special choice of the initial data $\mathbf{f}=\mathbf{R} /|\mathbf{R}|_{\mid z=1}$, for which the first $l$ components of the $\sigma(z)$-solution of (3.5a) - denoted here by $\sigma_{-}(z)$ - have a singularity of order $s,\left|\sigma_{-}(z)\right|=\vartheta(z-1)^{-s}$. Then, upon employing the equivalent $H$-norm, $\|\cdot\|_{H}^{2} \equiv \sum_{\nu=0}^{\infty}(\cdot, H \cdot)$ with $X_{-}^{*} H X_{-}=I$, one finds on account of (3.3), (3.5a) and (3.6c) that

$$
\|\psi\|^{2} \sim\|\psi\|_{H}^{2} \geqslant \text { const }\left.\cdot|z-1|^{-2 s} \cdot \sum_{\nu=0}^{\infty}\left|L_{-}^{\nu}(z) \sigma_{-} /\right| \sigma_{-}\right|^{2} ;
$$

we use (3.6b) to sum the term on the right-hand side, yielding the following lower bound

$$
\|\psi\|^{2} \geqslant \text { const } \cdot \lambda \cdot\left|\Lambda^{-}\right| \cdot|z-1|^{-(2 s+1)}
$$


Estimate (3.12b) can be also read as

$$
\left\|\left(z I-\mathcal{L}_{\Delta x}\right)^{-1}\right\| \geqslant \text { const } /|z-1|^{s+1 / 2}, \quad s \geqslant 1,
$$

which shows that the approximation fails to satisfy the resolvent estimate

$$
\left\|\left(z I-\varrho_{\Delta x}\right)^{-1}\right\| \leqslant \text { const } /(|z|-1),
$$

namely, it is unstable with respect to [5, Definition 3.2].*** Furthermore, the weaker $l_{2}$-instability follows as well. For if not, the approximation being $l_{2}$-stable [5, Definition 3.1] is characterized by having $\left\|\mathscr{L}_{\Delta x}^{n}\right\| \leqslant$ const which in turn leads to the contradiction that (3.14) holds for the geometric expansion of the resolvent $\left(z I-\varrho_{\Delta x}\right)^{-1}$. This completes the study of instability in the one-dimensional case.

Finally we remark about the multi-dimensional problem,

$$
\mathbf{P} \equiv A \frac{\partial}{\partial x}+\sum_{j=2}^{d} A^{(j)} \frac{\partial}{\partial x_{j}}+E, \quad\left(x_{2}, \ldots, x_{d}\right) \in \mathbf{R}^{d-1},
$$

approximated by (2.1) with the multi-indexes $\nu, j, r, p$ and $q$ varying appropriately. Denoting the tangential fourier dual variables by $\left(\omega_{2}, \ldots, \omega_{d}\right)$, the only point to note here is that, for $|z-1|+\sum_{j=2}^{d}\left|\omega_{j}\right|$ sufficiently small, the consistent normal block takes the form

$$
I+(\lambda A)^{-1} \cdot\left[(z-1) \cdot I+i \sum_{j=2}^{d} \omega_{j} A_{j}^{(j)}\right]
$$

by block diagonalization, its inflow (normal) part, $L_{-}$, see (3.6), can be separated from its outflow one. Once instability for sufficiently small tangential dual variables is established in the normal direction, instability is inferred back in the physical space. Q.E.D.

The rest of this section is concerned with an explicit estimate of the unstable growth indicated in the main theorem. We consider dissipative schemes, where we can restrict attention to the part of the discrete solution which corresponds to the resolvent $\psi(z)$ integrated in the neighborhood of $z=1$; see [9, Section 3]. Denoting that part by $v^{-}$and appealing to the same configuration as in $[9$, Section 3$]$, we have by (3.3) and (3.5a)

$$
v_{\nu}^{-}(t=n \cdot \Delta t)=\frac{1}{2 \pi i} \int_{\Gamma_{2}} z^{n} X(z) L^{\nu}(z) D^{-1}(z) d z,
$$

where the contour $\Gamma_{2}$ is given by

$$
\Gamma_{2}=\left\{z|| z-1|=\rho,| z \mid \geqslant 1-\varepsilon ; \rho \leqslant \rho_{0}, \varepsilon=\varepsilon\left(\rho_{0}\right)\right\} .
$$

To estimate $v_{\nu}^{-}(t)$ from below, we consider the previously chosen initial data $\mathbf{f}=\mathbf{R} / \mid \mathbf{R} \|_{z=1}$, for which the eigenprojection associated with $z=1$ has a pole of order $s$, see (3.10), and hence is of order

$$
\left|\frac{1}{2 \pi i} \int_{|z-1|=\rho} z^{n} X(z) L_{-}^{\nu}(z) \cdot(z-1)^{-s} \boldsymbol{\sigma}_{-} /\right| \boldsymbol{\sigma}_{-}|d z| \sim n^{s-1} .
$$

${ }^{* * *}$ In fact even the weaker stability [4, Definition 2.3] does not hold, since-using the notation of $[4$, Lemma 9.4$]$ - the necessary condition $\left|D_{22}^{-1}\right| \leqslant$ const is violated. 
When compared with (3.15), the last integral consists of integration of the resolvent along the extra path $\Gamma_{3}=\{z|| z-1|=\rho| z \mid, \leqslant 1-\varepsilon\}$; by Lemma 7 in [9], we can use the expansion (3.6b) along that extra path of integration, yielding

$$
\begin{aligned}
\mid \frac{1}{2 \pi i} \int_{\Gamma_{3}} z^{n} X_{-}(z) L_{-}^{\nu}(z) \cdot(z & -1)^{-s} \sigma_{-} /\left|\sigma_{-}\right| d z \mid \\
& \leqslant \text { const } \cdot\left(1-\frac{\rho}{\lambda \lambda_{l}^{-}}\right)^{\nu} \cdot \rho^{-(s-1)} .
\end{aligned}
$$

We note that by the finite speed of propagation, the last integral must cancel the source in (3.16) to at least exponentially small order, away from the boundary, $\nu \geqslant(n+1) \cdot r$. Near the boundary, however, $0<\nu<[\theta \cdot n]$, for appropriately chosen fixed $\theta$ and $K$ large enough so that $\exp \left(K \theta / \lambda\left|\lambda_{l}^{-}\right|\right) \cdot K^{-(s-1)}$ is sufficiently small, we find, with $\rho=K / n$, that after $n>K / \rho_{0}$ time-steps

$$
\left|v_{\nu}^{-}(t=n \cdot \Delta t)\right| \geqslant\left|\frac{1}{2 \pi i} \int_{|z-1|=\rho} \cdots d z\right|-\left|\frac{1}{2 \pi i} \int_{\Gamma_{3}} \cdots d z\right| \geqslant \text { const } \cdot n^{s-1}
$$

so we finally conclude

$$
\|v(t)\| \geqslant \text { const } \cdot\left[\sum_{\nu=0}^{[\theta \cdot n]}\left|v_{\nu}^{-}(t)\right|^{2}\right]^{1 / 2} \geqslant \text { const } \cdot\left[\frac{t}{\Delta t}\right]^{s-1 / 2}, \quad s \geqslant 1 .
$$

Thus, the discrete solution has a polynomial growth of order $s-1 / 2$, at least, which indeed corresponds to the singularity of order $s+1 / 2$ indicated in (3.13).

4. Examples and Additional Remarks. To demonstrate the strict sense of the stability definition 3.3 in [5], we consider the following example. The outflow scalar equation (1.1a) is approximated by a 3-point interior dissipative scheme whose boundary values are computed by temporal extrapolation

$$
v_{0}(t+\Delta t)=v_{0}(t)
$$

The overall approximation is clearly unstable with respect to [5, Definition 3.3] since the boundary values $\left\{v_{0}(t=n \cdot \Delta t)\right\}_{n=0}^{\infty}$ are computed in such a way that they are not $l_{2}$-integrable along the $t$-axis; indeed $z=1$ is an eigenvalue in this case, since the boundary determinant $-($ see $(3.8))-D(z)=z-1$ is vanishing at that point. Nevertheless, the overall approximation is $l_{2}$-stable [5, Definition 3.1] and convergent in this case, if we regard the computation of the boundary values as overspecification

$$
v_{0}(t)=f_{0},
$$

and apply [10, Theorem 1] (see also [15] for the multi-dimensional outflow problem). We note that both the stability and in particular the convergence (compare [3, Theorem 2.2]) are valid here in spite of having $z=1$ as an eigenvalue, the reason being that the eigenvalue $z=1$ is not originating from an inflow part of our outflow equation. That is, the power bounded Jordan cell $L$ in (3.3) contains no inflow modes and satisfies $|L(z=1)|<1$, which implies the standard damping away from the boundary. 
The above situation is in contrast to the undamped behavior in the inflow case where by $(3.6 a) L_{-}(z=1)=I$ : consider as an example the inflow scalar equation (1.1a) approximated by the dissipative Euler scheme

$$
v_{\nu}(t+\Delta t)=v_{\nu}(t)+\lambda a\left[v_{\nu}(t)-v_{\nu-1}(t)\right], \quad \nu=1,2, \ldots,-1<\lambda a<0,
$$

and augmented by linear horizontal extrapolation

$$
v_{0}(t+\Delta t)=v_{1}(t+\Delta t) \text {. }
$$

For vanishing interior initial data, $f_{\nu_{\nu>1}}=0$, one gets from (3.1a) and (3.3)

$$
v_{\nu}(t=n \cdot \Delta t)=\frac{1}{2 \pi i} \int_{\Gamma} z^{n}\left(\frac{\lambda a}{1+\lambda a-z}\right)^{\nu} \cdot \frac{1+\lambda a-z}{\lambda a \cdot(1-z)} f_{0} d z .
$$

The integral on the right consists of two contributions-the first is due to the undamped pole at $z=1$, yielding

$$
\frac{1}{2 \pi i} \int_{|z-1|=\rho} \cdots d z=f_{0}
$$

at all grid points $x_{\nu}$, which is cancelled out, as it should be, away from the boundary, by the other pole,

$$
\frac{1}{2 \pi i} \int_{|z-1-\lambda a|=\delta} \cdots d z=-f_{0}
$$

at $x_{\nu}, \nu \geqslant n+1$. Thus, we are left with the "boundary layer",

$$
v_{\nu}(t=n \cdot \Delta t)=\left[1-\sum_{k=0}^{\nu-1}\left(\begin{array}{l}
n \\
k
\end{array}\right) \cdot(-\lambda a)^{k} \cdot(1+\lambda a)^{n-k}\right] \cdot f_{0}, \quad 0 \leqslant \nu \leqslant n,
$$

any fixed portion of which amounts to the power-growth $\sim(t / \Delta t)^{1 / 2}$.

For a completely similar situation we refer the reader to the discussion of entropy violation by nonmonotone schemes in [6, Section 3]. By considering a steady shock as an interior boundary, it was shown there that the linearized Lax-Wendroff scheme may exhibit instability, the reason being exactly the zeroth-order extrapolation of an inflow-like quantity which yields the weakly unstable growth of order $\left\|T_{q}^{n}(b)\right\| \sim$ const $\cdot \sqrt{n}$; see $[6$, Section 3$]$.

Institute for Computer Applications in

Science and Engineering

NASA Langley Research Center

Hampton, Virginia 23665

1. A. BuRns, "A necessary condition for the stability of a difference approximation to a hyperbolic system of partial differential equations," Math. Comp., v. 32, 1978, pp. 707-724.

2. M. GOldBerg \& E. TADMOR, "Scheme-independent stability criteria for difference approximations of hyperbolic initial-boundary value problems. II," Math. Comp., v. 36, 1981, pp. 603-626.

3. B. GUSTAFSSON, "The convergence rate for difference approximations to mixed initial boundary value problems," Math. Comp., v. 29, 1975, pp. 396-406.

4. B. Gustafsson, H.-O. Kreiss \& A. Sundstrom, Stability Theory of Difference Approximations for Mixed Initial Boundary Value Problems. II, Report No. 30, Dept. of Comput. Sci., Uppsala Univ., Uppsala, Sweden, 1971.

5. B. GuSTAFSSON, H.-O. KREISS \& A. SUNDSTROM, "Stability theory of difference approximations for mixed initial boundary value problems. II," Math. Comp., v. 26, 1972, pp. 649-686.

6. A. Harten, J. M. Hyman \& P. D. Lax, "On finite difference approximations and entropy conditions for shocks,” Comm. Pure Appl. Math., v. 29, 1976, pp. 297-322. 
7. R. HerSH, “Mixed problems in several variables," J. Math. Mech., v. 12, 1963, pp. 317-334.

8. H.-O. KREISS, "Difference approximations for hyperbolic differential equations," Numerical Solution of Partial Differential Equations (Proc. Sympos. Univ. of Maryland, 1965), Academic Press, New York, 1966, pp. 51-58.

9. H.-O. KREISs, "Stability theory for difference approximations of mixed initial boundary value problems. I," Math. Comp., v. 22, 1968, pp. 703-714.

10. H.-O. KREISS \& E. LundQuist, "On difference approximations with wrong boundary values," Math. Comp., v. 22, 1968, pp. 1-12.

11. H.-O. Kreiss \& J. Oliger, Methods for the Approximate Solution of Time Dependent Problems, GARP Publication Series No. 10, 1973.

12. P. LANCASTER, "A fundamental theorm on lambda-matrices with applications. II. Finite difference equations," Linear Algebra Appl., v. 18, 1977, pp. 213-222.

13. E. TADMOR, Scheme-Independent Stability Criteria for Difference Approximations to Hyperbolic Initial-Boundary Value Systems, Ph.D. thesis, Dept. of Math. Sci., Tel-Aviv Univ., December 1978.

14. L. Trefethen, Group Velocity in Finite Difference Schemes, Report No. NA-81-12, Dept. of Comput. Sci., Stanford Univ., Stanford, 1981.

15. S. OSHER, "On systems of difference equations with wrong boundary conditions," Math. Comp., v. 23, 1969, pp. $567-572$. 